\title{
On the spatial metric of short-SOA costs of exogenous cuing
}

\author{
PEGGY CHEN, CATHLEEN MOORE, AND J. TOBY MORDKOFF \\ Penn State University
}

\begin{abstract}
When the stimulus onset asynchrony (SOA) between the cue and the target is short, exogenous spatial cues usually produce a response time benefit. However, consistent with several recent studies, we have found that a short stimulus onset asynchrony is not sufficient. At least one more factor-the number of cue and target locations-also plays a role. Even more interesting, when 8 cue and target locations are used, the effect of an exogenous cue produces a cost on valid cue trials, and the spatial metric of this negative cuing effect depends on whether the cue remains visible at target onset.
\end{abstract}

It has long been known that certain salient events can evoke an involuntary shift of covert visual attention. Based on early work by Posner and Cohen (e.g., Posner \& Cohen, 1984), a standard paradigm for studying these shifts has been developed. The task requires a simple detection response to a visual target presented away from fixation. Shortly before the target presentation, an uninformative cue (i.e., a stimulus whose location is uncorrelated with the location of the target) appears in one of the possible target locations. Typically, responses to targets in the same location as the cue are faster than responses to targets presented elsewhere, at least when the cueto-target stimulus onset asynchrony (SOA) is short (e.g., less than $200 \mathrm{~ms}$ ). The standard explanation for this spatial cuing effect is that covert visual attention is summoned to the location of the salient cue. This summoning or capture of attention facilitates the processing of any item that appears in the same location and, conversely, interferes with the processing of distant items (for a recent review, see Prinzmetal, McCool, \& Park, 2005).

The nature of exogenous orienting has been studied intensively with the Posner cuing paradigm, a simple detection task with an uninformative peripheral cue. (For a demonstration of the effects using more complicated tasks, see Lupiáñez, Milliken, Solano, Weaver, \& Tipper, 2001). A large part of this research has been aimed at disentangling the mechanisms that underlie the biphasic facilitation and inhibition that are observed at various SOAs between the cue and the target (McAuliffe \& Pratt, 2005; Tassinari, Aglioti, Chelazzi, Peru, \& Berlucchi, 1994). The emergent hypothesis 
that facilitation and inhibition are produced by separate mechanisms has been supported by most studies (for a review, see Klein, 2000), with facilitation being earlier and more transient than inhibition. Thus, it is widely accepted that a short SOA is needed to find a benefit of exogenous cuing, caused by an involuntary shift of visual attention.

However, beneficial cuing effects with short SOAs are not as robust as once thought. That is, an exogenous cue does not always facilitate responses to targets in the cued location, even with short SOAs. For example, Tassinari and colleagues repeatedly failed to observe response time (RT) benefits at the cued location using SOAs as short as $65 \mathrm{~ms}$ (see Tassinari \& Berlucchi, 1995). This raises questions about the biphasic nature of the cuing effects by suggesting that inhibition does not necessarily follow facilitation. Possibly other criteria, in addition to a short SOA, are necessary to produce a positive cuing effect. Across studies using short SOAs, a variety of variables have been examined, including cue-target temporal overlap (Berger, Dori, \& Henik, 1999; Maruff, Yucel, Danckert, Stuart, \& Currie, 1999; McAuliffe \& Pratt, 2005), stimulus eccentricity (Berger et al., 1999; Berlucchi, Tassinari, Marzi, \& Stefano, 1989; Collie, Maruff, Yucel, Danckert, \& Currie, 2000), cue-target physical similarity (McAuliffe \& Pratt, 2005; Pratt, Hillis, \& Gold, 2001), and the number of cue and target locations (Gawryszewski, Carreiro, \& Magalhães, 2005; Wu \& Remington, 2005). To summarize the implications of this work, in order to maximize the chances of observing a benefit of cuing with a short SOA, the cue should not appear in the exact same location as the target (e.g., the cue should involve a surrounding placeholder), the cue should remain on the screen when the target appears, and the number of cue and target locations should be less than eight.

Recently, independent groups have reported short-SOA costs with eight cue and target locations (Chen, Mordkoff, \& Moore, 2005; Wu \& Remington, 2005 ). Likewise, Gawryszewski et al. (2005) published a negative cuing effect with eight locations. Because most of the previous studies that found positive cuing effects used only two cue and target locations, there is clearly a need to directly compare these conditions: one in which there are two cue and target locations and another with eight. At the same time, it is worth noting that both Chen et al. and Gawryszewski et al. did not use the optimal condition for obtaining a positive cuing effect: a cue that overlapped the target in time (see Collie et al., 2000; Maruff et al., 1999). In contrast, Wu and Remington did manipulate cue-target temporal overlap and found that when the cue remained on at target onset, there was a trend towards positive cuing at one SOA, but it was not the shortest SOA and it was not statistically significant. Because of these inconsistent findings across experiments, along with several other uncontrolled differences, the present experiment directly manipulated 
the number of cue and target locations and the temporal overlap between the cue and target, with all else held constant.

Of course, the use of large numbers of cue and target locations (in at least some conditions) raises another issue that should be addressed. With more than two locations, the separation between the cue and target locations can vary across invalid cue trials. For example, Gawryszewski et al. (2005) found that responses were faster (on invalid cue trials) as the distance between the cue and target increased. But their method confounded two different metrics for assessing this effect: the linear distance between the cue and target and the angular distance (as subtended at fixation). In order to determine which of these metrics is responsible for the observed pattern of results, the present experiment also included a manipulation of display eccentricity. Increasing the eccentricity has a direct effect on the linear distance between two locations but has no effect on the angular distance. By manipulating the eccentricity of the cue and target locations, therefore, we could explore the spatial characteristic of the effects that are found with large numbers of cue and target locations.

\section{EXPERIMENT}

To ensure that it was in the very short range, the SOA was fixed at $50 \mathrm{~ms}$. The experiment included manipulations of cue-target temporal overlap (i.e., whether the cue remained visible when the target appeared), number of cue and target locations, and eccentricity of these locations from fixation. With a fixed duration for the SOA $(50 \mathrm{~ms})$, two durations for the cue were used (100 and $50 \mathrm{~ms}$ ), such that one condition produced temporal overlap and the other condition did not. With regard to the number of cue and target locations, half of the trials involved just two locations, directly to the left and right of fixation (as is typical), and half involved eight locations arranged on an imaginary circle around fixation (Figure 1). In the case of eccentricity (i.e., the distance from fixation to the ring of locations at which cues and targets could appear) a range of values were used that span those that have become popular $(3,5$, and 7 degrees of visual angle [dva]).

\section{METHOD}

\section{Participants}

Eighty participants were recruited from the Pennsylvania State University undergraduate participant pool. All participants reported normal or corrected-to-normal visual acuity and full color vision, and all were naive as to the purpose of the experiment. Participants received partial credit in an introductory course in psychology. 


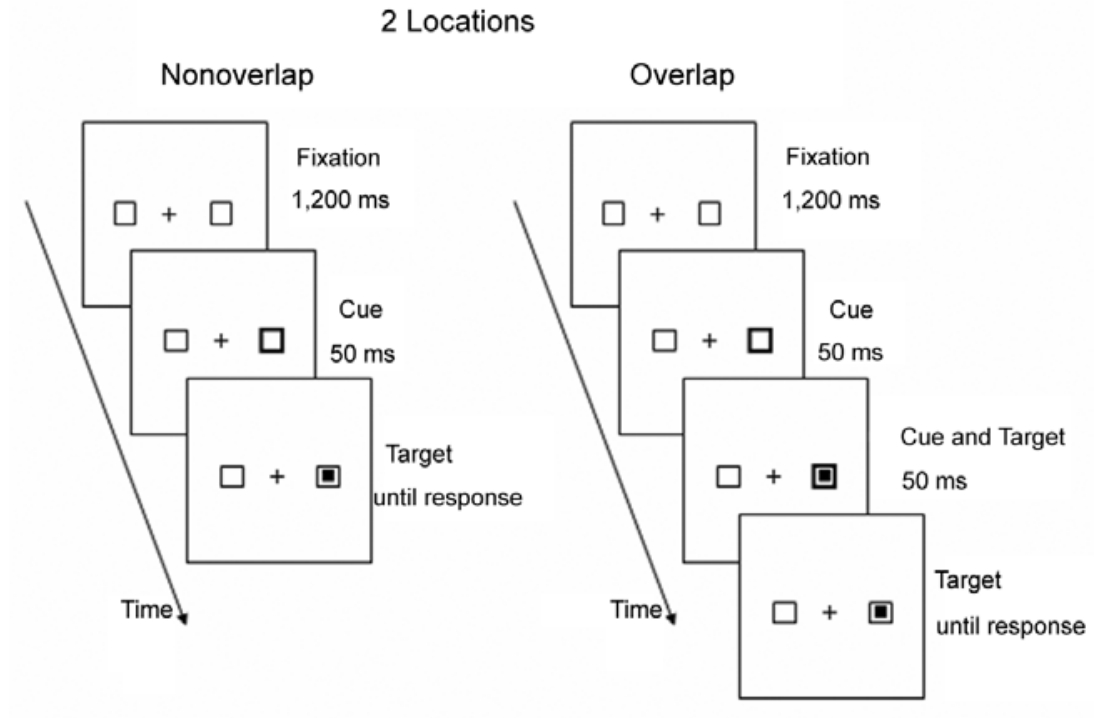

8 Locations
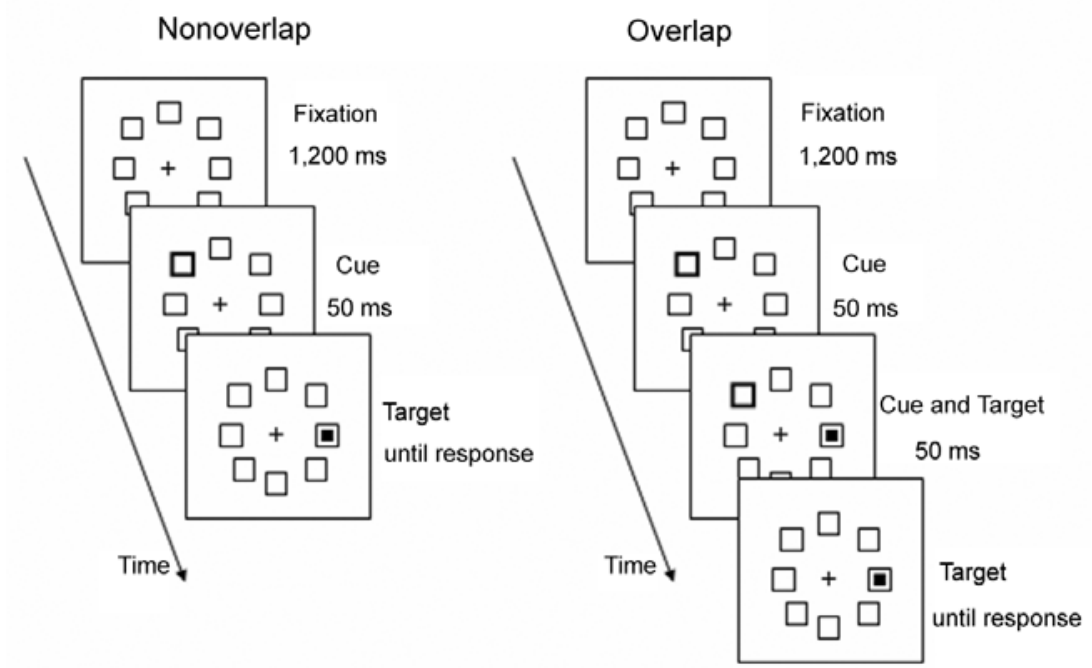

Figure 1. Trial sequences for 2 and 8 locations of cue and target

\section{Apparatus}

The experiment took place in a dimly lit room. The stimuli were presented on a 15-inch color monitor, viewed from a distance of approximately $56 \mathrm{~cm}$, with a refresh rate of $60 \mathrm{~Hz}$. The trial events and data collection were controlled by an IBM-compatible, Pentium-based personal computer, programmed in Turbo Pascal 7.0. 


\section{Stimuli}

Each trial began with the presentation of a fixation display. This included a central cross and either two or eight square placeholders. The fixation cross was $0.18 \mathrm{dva}$ in height and width. The eccentricities of the placeholders, cues, and targets were all 3, 5, or 7 dva, depending on condition. A cue was presented as brightening of one of the placeholders (by changing the VGA color code from 7 to 15). The target was a filled square inside one of the placeholders. To equate visibility across the three eccentricities, placeholder, cue, and target size were both adjusted (Anstis, 1974). On 3-dva trials, the placeholders and cues were $0.35 \mathrm{dva}$ and the target was $0.20 \mathrm{dva}$; on 5-dva trials, they were 0.61 and $0.41 \mathrm{dva}$, respectively; on 7-dva trials, they were 1.01 and $0.69 \mathrm{dva}$, respectively. When two locations were used, only the 3 and 9 o'clock locations were used. When eight were used, the placeholders (and subsequent cues and targets) appeared at 12:00, 1:30, 3:00, 4:30, 6:00, 7:30, 9:00, and 10:30.

\section{Procedure}

A $2 \times 2 \times 3 \times 2$ design was used, including two between-participant factors (temporal overlap and number of locations, two vs. eight) and two within-participant factors (eccentricity, with circle radii of 3, 5, and 7 dva in separate blocks, and validity). Nested within the number of locations was a fifth factor that concerned the distance (on invalid cue trials) between the cue and the target. In particular, when there were eight display locations, the target could appear $45^{\circ}, 90^{\circ}, 135^{\circ}$, or $180^{\circ}$ away from the cue on invalid cue trials (see the lower panel of Figure 1 for an example of $135^{\circ}$ ).

The cue did not provide any information about target location because the cue and target locations were selected at random on every trial. Thus, half of the trials with two locations were valid, whereas only one-eighth of the trials with eight locations were valid. The three values of eccentricity occurred equally often within a block of trials.

After a block of 15 trials, participants went on to complete 15 full blocks of about 30 trials in a single 1-hr session. The participants were required to press a button with the index finger on their favored hand as quickly as possible when a target was shown. The target remained visible until a response was made. The proportion of catch trials (i.e., trials without a target) was $11 \%$. If no response was detected within 2,050 ms of cue onset (i.e., 2,000 ms after the time when a target would have been shown), the screen went blank. If an error was made, feedback was given for $3,500 \mathrm{~ms}$. The next trial started after an intertrial interval of 1,000 ms. The participants were asked to make their responses as quickly as possible while maintaining at least $95 \%$ accuracy.

\section{RESULTS}

The short block and the first three full blocks were considered practice blocks and not included in the analyses. Only RTs for correct responses were analyzed (Table 1 ). The initial analysis followed the $2 \times 2 \times 3 \times 2$ 
design described earlier. As shown in Figure 2, a strong interaction was found between validity and the number of locations, $F(1,76)=23.96$, $p<.001$. The apparent three-way interaction between validity, number of locations, and temporal overlap was not reliable, $F<1.00$. The main effect of temporal overlap approached significance, $F(1,76)=3.91, p=.052$. The number of locations did not yield a significant main effect, and the two between-participant factors did not interact. As to the within-participant manipulations, both eccentricity and validity were reliable, $F(2$, $152)=15.73, p<.001$, and $F(1,76)=6.17, p=.015$, respectively. No other main effects or interactions were found.

To explore the observed interaction between validity and the number of locations, separate analyses were conducted as a function of the number of cue and target locations. For two locations, there was only a trend toward a cuing benefit, $F(1,38)=2.88, p<.10$. In contrast, responses were reliably slower when the cue and target did not overlap, $F(1,38)=4.43$, $p=.042$, and mean RT was greater for the smaller displays, $F(2,76)=6.54$, $p=.002$. No interactions were found.

For eight locations, there was a large and reliable negative cuing effect, with responses on valid cue trials being slower, $F(1,38)=27.44, p<.001$. Temporal overlap did not produce a main effect, but the same effect of eccentricity as for two locations was observed, $F(2,76)=9.34, p<.001$. Also as before, there were no interactions.

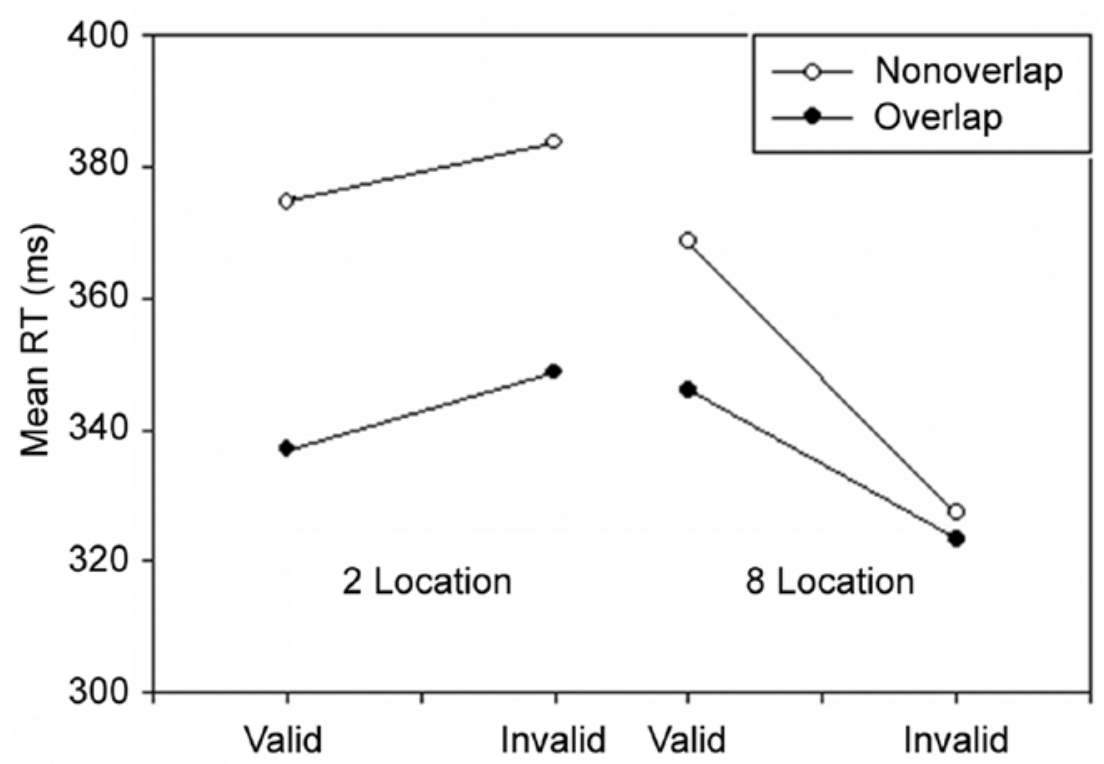

Figure 2. Mean RT as a function of the number of cue and target locations, cuetarget temporal overlap, and cuing validity 


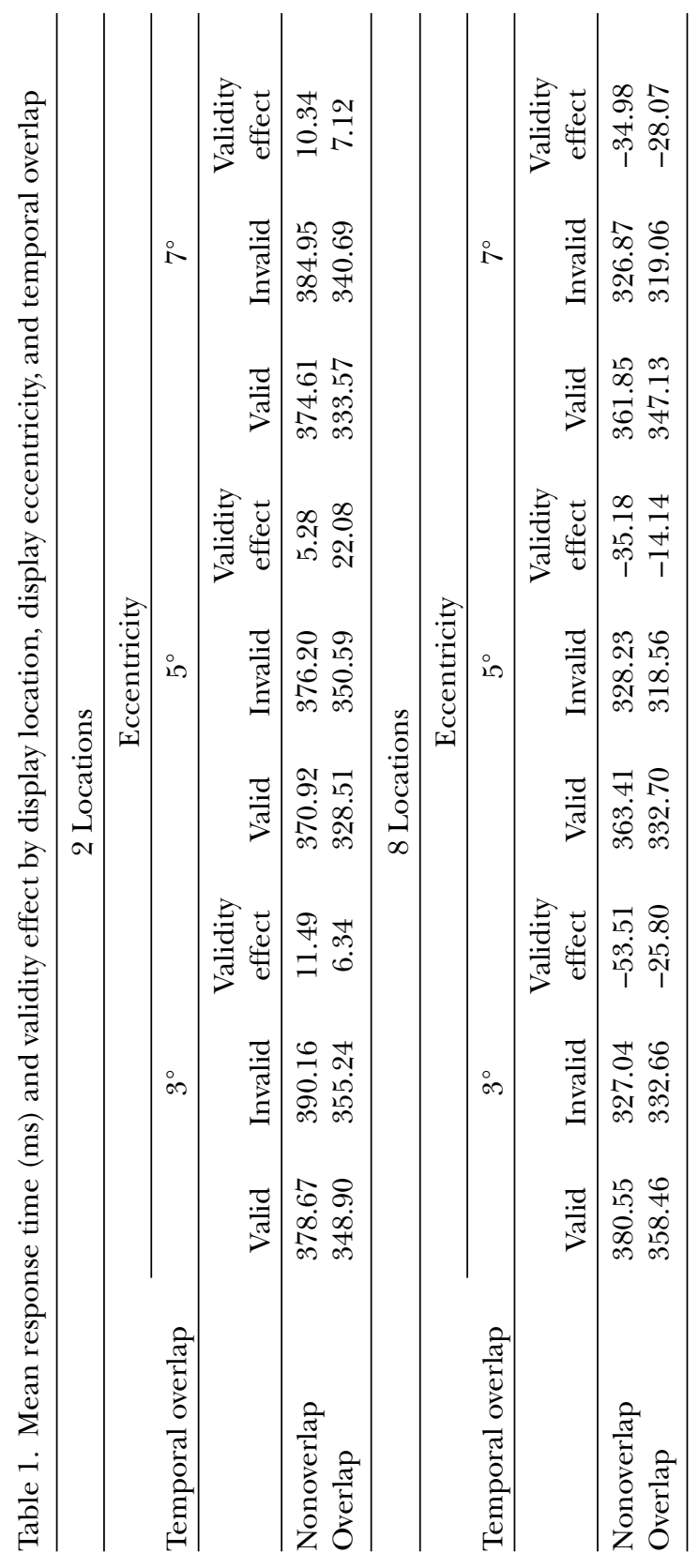


The final set of analyses concerned the spatial metric that underlies the negative cuing effect observed with eight cue and target locations. Two possibilities were tested: The "benefit" of an invalid cue depends on the straight-line distance between the cue and the target, or the "benefit" depends on the angular distance (as defined by the angle, subtended at fixation, between the cue and target locations). The former, linear metric takes eccentricity into account. The latter, angular metric does not. We tested these models by fitting the data, using the jackknife procedure (Cornfield \& Tukey, 1956). Although there is some debate whether jackknifing is appropriate under these conditions (Ashby, Maddox, \& Lee, 1994; Nosofsky, 1991), the limited number of observations (per participant) made this necessary in order to get stable estimates of dispersion at a variety of points along each of the curves defined by the two spatial metrics.

A $2 \times 2$ mixed-factor analysis of variance was conducted on the root mean squared errors (RMSEs) as a function of whether the cue and the target overlapped in time and the type of model (linear distance vs. angular distance) used. The interaction between temporal overlap and model was highly significant (even after correction for jackknifed data), $F(1$, $38)=22.92, p<.001$. Therefore, separate pairwise tests were conducted for each level of temporal overlap. When the cue was removed at target onset, the angular model provided the better fit (mean RMSEs $=3.32$ and 4.82 for the angular and linear models, respectively), $t(19)=2.64$, $p<.05$. In contrast, when the cue remained visible after target onset, the linear model was supported (mean RMSEs $=8.35$ and 6.46 for angular and linear), $t(19)=4.49, p<.001$.

\section{DISGUSSION}

In contrast to what might be inferred from the early literature (e.g., Posner \& Cohen, 1984), a short SOA is not sufficient to produce a positive benefit of exogenous cuing. The present experiment joins several others in failing to find a significant positive cuing effect, even with only two cue and target locations. Furthermore, these results make it clear that whether the cues overlap the target in time does not determine the sign of cuing effects. As shown in Figure 2, for example, whether the cue and the target overlapped or not, cuing did not (significantly) affect RT when there were two stimulus locations, and cued targets produced slower responses than uncued targets when there were eight locations. Thus, these data show that inhibition can be found as early as $50 \mathrm{~ms}$ after cue onset, which is inconsistent with the idea that inhibition always follows facilitation (Berger et al., 1999; McAuliffe \& Pratt, 2005). Instead of temporal overlap, as suggested by Maruff et al. (1999), the number of cue and target locations 
appears to be the key. Therefore, it is recommended that large displays be avoided if the goal is to observe a positive cuing effect.

Interestingly, we also found that the negative cuing effect is greatest in the locations that are closer to the cue and gradually lessens as the target is placed farther away. To gain a better understanding of this inhibitory effect of spatial cuing, we tested the following hypothesis. Most of all, a peripheral, uninformative cue generates inhibitory effects. This cue-induced inhibition includes two components: One is sensory and resembles masking, whereas the other is oculomotor, probably as a result of the requirement to maintain fixation. Physiologic evidence shows that early in the visual system, such as within the lateral geniculate nucleus, and in the striate and extrastriate visual cortex, the structure of receptive fields linearly represents the spatial arrangement of visual stimuli (for reviews, see DeAngelis, Ohzawa, \& Freeman, 1995; Gattass et al., 2005). Sensory competition between adjacent stimuli has also been shown in retinotopic brain areas (Schwartz et al., 2005). Thus, applying the physiologic findings to our working hypothesis, the inhibitory effect produced by the sensory component would spread linearly from the cue location; that is, the strength of this inhibition would gradually decrease as the distance from the cue increases.

In contrast, the inhibition caused by the oculomotor component would depend on a spatial metric whose reference point is eye fixation instead of the cue. To inhibit an eye movement, one has to suppress both aspects, direction and amplitude, of the saccadic vector. Before signals are sent to the saccade generators in the brain stem (see Sparks, 2002), certain neurons in the frontal eye fields, the supplementary eye fields, and the superior colliculus are activated in order to determine the direction and amplitude of the saccade (Bruce, Friedman, Kraus, \& Stanton, 2004; Bruce \& Goldberg, 1985; Lee, Rohrer, \& Sparks, 1988; Russo \& Bruce, 2000). It has been shown that the polar direction and the amplitude of a given saccade are coded separately, with different combinations of neuronal populations' activity and duration of said activity. Thus, it is possible to observe a behavioral effect that reflects one coordinate (direction or amplitude) of a saccade. In the present experiment, when a cue appeared, a saccade toward the cue would be inhibited, followed by another inhibition of eye movement toward the subsequent target. In each trial, the cue and the target were in the same eccentricity. Thus, the inhibition of eye movement toward the two stimuli would differ in direction but not in amplitude, meaning that the angle between the cue and the target (as subtended at fixation), rather than eccentricity, would have been revealed as a critical factor for the inhibitory effect.

The two components are not mutually exclusive but can be more or 
less involved, depending on the situation. Temporal overlap between the cue and target may help to determine whether the sensory or oculomotor component plays the stronger role. When the cue remains visible at the target onset, the sensory component would be emphasized because of the potential for masking between the two stimuli. In contrast, when the cue is removed at the target onset, the occurrence of the two distinct events would emphasize the oculomotor component. That is, if the aforementioned two-component model of inhibition is correct, one should find that the inhibitory effect depends more on the linear distance when the cue and target overlap in time and more on the angular distance when the cue and target do not overlap. This is exactly what our results showed: The negative cuing effect with overlapping cues and targets depended more on the linear distance than the angular distance, which indicates more involvement of the sensory component of inhibition; the negative cuing effect found in the nonoverlap condition depended more on the polar coordinates, which suggests more influence from the oculomotor component.

In closing, it is useful to note that the negative effects of spatial cues with long SOAs (i.e., cue-target intervals of $200 \mathrm{~ms}$ or more) have also been hypothesized to arise in both sensory and motor-related processes (see Berlucchi, 2006; Klein, 2000). However, this parallel should not be read as a claim that the same mechanisms are responsible for all the effects found at both short and long SOAs because there is ample evidence that this is unlikely. For example, it would be very difficult to explain short-SOA inhibition in terms of "attentional momentum," which is one possibility for long SOAs (Pratt, Adam, \& McAuliffe, 1998; Pratt, Spalek, \& Bradshaw, 1999), because $50 \mathrm{~ms}$ is clearly insufficient for both the capture and the release of visual attention. Therefore, further investigation will be needed to disentangle the short- and long-SOA effects of exogenous cues.

\section{Notes}

Correspondence about this article should be addressed to Peii (Peggy) Chen, University of Medicine and Dentistry of New Jersey, Department of Physical Medicine and Rehabilitation, Kessler Medical Rehabilitation Research and Education Center, 1199 Pleasant Valley Way, West Orange, NJ 07052 (e-mail: pchen@kmrrec.org). Received for publication August 4, 2006; revision received November 2006.

\section{References}

Anstis, S. M. (1974). A chart demonstrating variation in acuity with retinal position. Vision Research, 14, 589-592.

Ashby, F. G., Maddox, W. T., \& Lee, W. W. (1994). On the dangers of averaging across subjects when using multidimensional-scaling or the similarity-choice model. Psychological Science, 5, 144-151.

Berger, A., Dori, H., \& Henik, A. (1999). Peripheral non-informative cues do 
induce early facilitation of target detection. European Journal of Cognitive Psychology, 11, 119-137.

Berlucchi, G. (2006). Inhibition of return: A phenomenon in search of a mechanism and a better name. Cognitive Neuropsychology, 23, 1065-1074.

Berlucchi, G., Tassinari, G., Marzi, C. A., \& Stefano, M. D. (1989). Spatial distribution of the inhibitory effect of peripheral non-informative cues on simple reaction time to non-fixated visual targets. Neuropsychologia, 27, 201-221.

Bruce, C. J., Friedman, H. R., Kraus, M. S., \& Stanton, G. B. (2004). The primate frontal eye field. In L. M. Chalupa \& J. S. Werner (Eds.), The visual neurosciences (Vol. 2). Cambridge, MA: MIT Press.

Bruce, C. J., \& Goldberg, M. E. (1985). Primate frontal eye fields. 1. Single neurons discharging before saccades. Journal of Neurophysiology, 53, 603-635.

Chen, P., Mordkoff, J. T., \& Moore, C. M. (2005). Responding to the second of two events: The farther away, the better. Poster session presented at the 5th Annual Meeting of the Vision Sciences Society, Sarasota, FL.

Collie, A., Maruff, P., Yucel, M., Danckert, J., \& Currie, J. (2000). Spatiotemporal distribution of facilitation and inhibition of return arising from the reflexive orienting of cover attention. Journal of Experimental Psychology: Human Perception and Performance, 26, 1733-1745.

Cornfield, J., \& Tukey, J. W. (1956). Average values of mean squares in factorials. Annals of Mathematical Statistics, 27, 907-949.

DeAngelis, G. C., Ohzawa, I., \& Freeman, R. D. (1995). Receptive-field dynamics in the central visual pathways. Trends in Neurosciences, 18, 451-458.

Gattass, R., Nascimento-Silva, S., Soares, J. G. M., Lima, B., Jansen, A. K., Diogo, A. C. M., et al. (2005) . Cortical visual areas in monkeys: Location, topography, connections, columns, plasticity and cortical dynamics. Philosophical Transactions of the Royal Society B: Biological Sciences, 360, 709-731.

Gawryszewski, L. G., Carreiro, L. R. R., \& Magalhães, F. V. (2005). Early and late inhibitions elicited by a peripheral visual cue on manual response to a visual target: Are they based on Cartesian coordinates? Psicológica, 26, 121-137.

Klein, R. M. (2000). Inhibition of return. Trends in Cognitive Sciences, 4, 138-147.

Lee, C. K., Rohrer, W. H., \& Sparks, D. L. (1988). Population coding of saccadic eye-movements by neurons in the superior colliculus. Nature, 332, 357-360.

Lupiáñez, J., Milliken, B., Solano, C., Weaver, B., \& Tipper, S. P. (2001). On the strategic modulation of the time course of facilitation and inhibition of return. Quarterly Journal of Experimental Psychology, 54A, 753-773.

Maruff, P., Yucel, M., Danckert, J., Stuart, G., \& Currie, J. (1999). Facilitation and inhibition arising from the exogenous orienting of covert attention depends on the temporal properties of spatial cues and targets. Neuropsychologia, 37, $731-744$.

McAuliffe, J., \& Pratt, J. (2005). The role of temporal and spatial factors in the covert orienting of visual attention tasks. Psychological Research, 69, 285-291.

Nosofsky, R. M. (1991). Tests of an exemplar model for relating perceptual classification and recognition memory. Journal of Experimental Psychology: Human Perception and Performance, 17, 3-27. 
Posner, M. I., \& Cohen, Y. A. (1984). Components of visual orienting. In H. Bouma \& D. G. Bouwhuis (Eds.), Attention $\mathcal{E}$ performance X. Hillsdale, NJ: Erlbaum. Pratt, J., Adam, J. J., \& McAuliffe, J. (1998). The spatial relationship between cues and targets mediates inhibition of return. Canadian Journal of Experimental Psychology, 52, 213-216.

Pratt, J., Hillis, J., \& Gold, J. M. (2001). The effect of the physical characteristics of cues and targets on facilitation and inhibition. Psychonomic Bulletin E Review, 8, 489-495.

Pratt, J., Spalek, T. M., \& Bradshaw, F. (1999). The time to detect targets at inhibited and noninhibited locations: Preliminary evidence for attentional momentum. Journal of Experimental Psychology: Human Perception and Performance, 3, 730-746.

Prinzmetal, W., McCool, C., \& Park, S. (2005). Attention: Reaction time and accuracy reveal different mechanisms. Journal of Experimental Psychology: General, 134, 73-92.

Russo, G. S., \& Bruce, C. J. (2000). Supplementary eye field: Representation of saccades and relationship between neural response fields and elicited eye movements. Journal of Neurophysiology, 84, 2605-2621.

Schwartz, S., Vuilleumier, P., Hutton, C., Maravita, A., Dolan, R. J., \& Driver, J. (2005). Attentional load and sensory competition in human vision: Modulation of fMRI responses by load at fixation during task-irrelevant stimulation in the peripheral visual field. Cerebral Cortex, 15, 770-786.

Sparks, D. L. (2002). The brainstem control of saccadic eye movements. Nature Reviews: Neuroscience, 3, 952-964.

Tassinari, G., Aglioti, S., Chelazzi, L., Peru, A., \& Berlucchi, G. (1994). Do peripheral non-informative cues induce early facilitation of target detection? Vision Research, 34, 179-189.

Tassinari, G., \& Berlucchi, G. (1995). Cover orienting to non-informative cues: Reaction time studies. Behavioural Brain Research, 71, 101-112.

Wu, S. C., \& Remington, R. W. (2005). The parting shot: Effects of offsets on attentional orienting. Poster session presented at the 46th annual meeting of the Psychonomic Society, Toronto, Ontario, Canada. 